\title{
COUPLING INTERACTION BETWEEN THE POWER COUPLER AND THE THIRD HARMONIC SUPERCONDUCTING CAVITY*
}

\author{
Jianjian Li, Nikolay Solyak", FNAL, Batavia, IL 60510, U.S.A. \\ Thomas Wong, IIT, Chicago, IL 60616, U.S.A.
}

\begin{abstract}
Fermilab has developed a third harmonic superconducting cavity operating at the frequency of 3.9 $\mathrm{GHz}$ to improve the beam performance for the FLASH user facility at DESY. It is interesting to investigate the coupling interaction between the SRF cavity and the power coupler with or without beam loading. The coupling of the power coupler to the cavity needs to be determined to minimize the power consumption and guarantee the best performance for a given beam current. In this paper, we build and analyze an equivalent circuit model containing a series of lumped elements to represent the resonant system. An analytic solution of the required power from the generator as a function of the system parameters has also been given based on a vector diagram.
\end{abstract}

\section{INTRODUCTION}

Third harmonic superconducting cavities shown in Fig. 1 are proposed to increase the peak bunch current of the beam and to linearize the accelerating voltage (energy distribution) acting on the electrons within a bunch in the longitudinal phase space for the FLASH user facility at DESY [1]. The third harmonic cryomodule will be installed downstream of the first accelerating module, containing $1.3 \mathrm{GHz}$ TESLA superconducting cavities.

The first four cavities were completed. Lessons and experiences were learned from the design, construction, and cryogenic test of those cavities. Cavity five and six are on schedule and currently under test. Parts for the remaining two cavities are in hand, and fabrication is in good progress. The cavity design parameters are shown in Table 1. Up to $23 \mathrm{MV} / \mathrm{m}$ accelerating gradient in pulsed mode has been achieved from the cryogenic test of cavity four in a vertical test stand at Fermilab. More information regarding the test can be found in [2] and [3].

A new $3.9 \mathrm{GHz}$ power coupler for the third harmonic cavities has been developed at Fermilab after a series of parameter optimizations and calculations [4]. The power coupler is shown in Fig. 2. Design parameters of the power coupler are shown in Table 2. The first two power couplers have been assembled in a test stand and under high power processing at Fermilab. With the pulse length of $1300 \mu \mathrm{s}$ and the repetition period of $3 s$, around 60 $\mathrm{kW}$ RF power has been successfully fed into the coupler test stand without any problems before the klystron begins to saturate. Details of the coupler test can be found in [5].

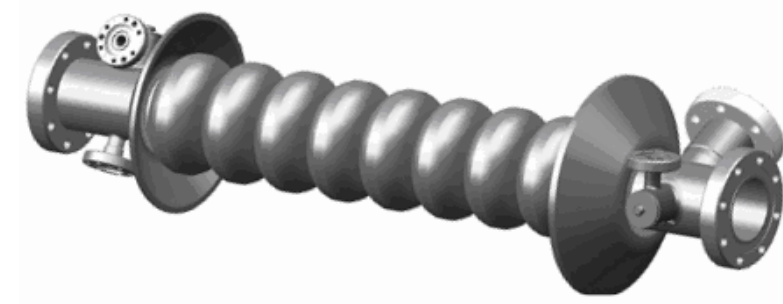

Figure 1: A naked third harmonic superconducting cavity.

Table 1: Design parameters of the third harmonic cavity.

\begin{tabular}{|l|l|}
\hline \multicolumn{2}{|c|}{ Design parameters of the third harmonic cavity } \\
\hline Accelerating Mode & $\pi$ mode \\
\hline Frequency & $3.9 \mathrm{GHz}$ \\
\hline Active Length & $0.3459 \mathrm{~m}$ \\
\hline Number of Cells & 9 \\
\hline Phase & $-179 \mathrm{deg}$. \\
\hline$R / Q$ & $375 \mathrm{Ohm}$ \\
\hline Beam Current & $9 \mathrm{~mA}$ \\
\hline Accelerating Gradient & $14.5 \mathrm{MV} / \mathrm{m}$ \\
\hline Qext for Accelerating Mode & $9.5 \mathrm{e}+5$ \\
\hline BBU Limit for HOM, Q & $<1.0 \mathrm{e}+5$ \\
\hline
\end{tabular}

Figure 2: The $3.9 \mathrm{GHz}$ power coupler.

Table 2: Main features of the power coupler.

\begin{tabular}{|l|l|}
\hline \multicolumn{2}{|c|}{ Main features of the power coupler } \\
\hline Frequency & $3.9 \mathrm{GHz}$ \\
\hline Pulse Length & $\begin{array}{l}1.3 \mathrm{~ms}(0.5 \mathrm{~ms} \text { rise time and } 0.8 \\
\mathrm{ms} \text { flat top coincident with beam } \\
\text { current) }\end{array}$ \\
\hline Rep. Rate & $5 \mathrm{~Hz}$ \\
\hline Peak Power & $45 \mathrm{~kW} \mathrm{(9} \mathrm{mA}$ Beam Current) \\
\hline Type & Coaxial \\
\hline Windows & $\begin{array}{l}\text { Cylindrical and Planar (TiN Coated } \\
\text { on Vacuum Side) }\end{array}$ \\
\hline Impedance & 50 Ohm (6.5 mm/15 mm) \\
\hline Bellows & Two \\
\hline Antenna & Hollow Stainless Steel (Cu Plated) \\
\hline
\end{tabular}


The loaded quality factor is a very important parameter which needs to be set to couple proper amount of power from the generator to the cavity and the beam. The loaded quality factor as a function of the antenna penetration length calculated from a nine-cell cavity model in HFSS is shown in Fig. 3. When the antenna is flush with the wall of the beam tube, a loaded quality factor of $7.6 \mathrm{e}+5$ is obtained. A higher coupling value can also be reached by proper adjustment of the antenna penetration length.

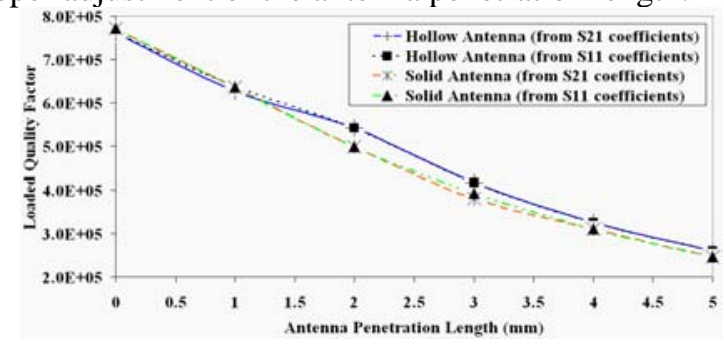

Figure 3: The loaded quality factor versus antenna penetration length.

\section{MODEL OF THE COUPLING SYSTEM}

\section{No Beam Injection}

The coupling system without beam injection is shown in Fig. 4. Between the klystron and the power coupler is an isolator which ensures that power coming from the cavity is terminated in a matched load. It is necessary since the klystron may be destroyed by the reflected waves.

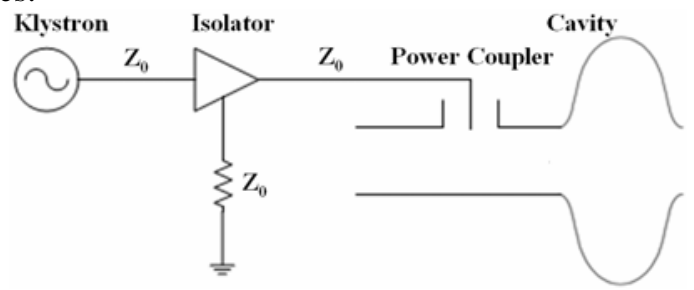

Figure 4: Model of the cavity coupled to the klystron via a power coupler.

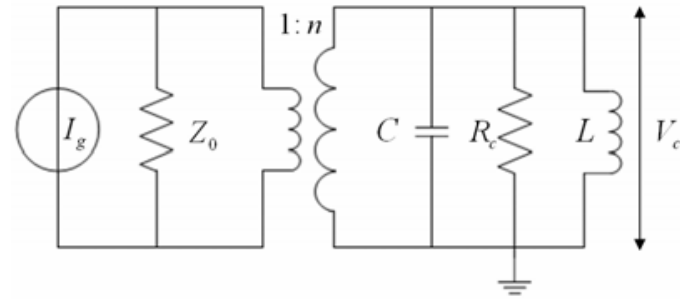

Figure 5: Equivalent circuit model of the coupling system.

The equivalent circuit model is shown in Fig. 5. The klystron and isolator combination is modeled by an ideal current source with a shunt resistor. Resonant mode in the cavity can be equivalently described by means of a parallel RLC circuit [6]. The cavity voltage $V_{c}$ is defined as the maximum accelerating voltage acting on a particle. The power coupler is modeled by a transformer in the ratio of 1 to $\mathrm{n}$. All calculations are derived from the following differential equation based on Kirchhoff current law [7]:
$C \frac{d V_{c}(\omega, t)}{d t}+\frac{1}{L} \int V_{c}(\omega, t) d t+\frac{V_{c}(\omega, t)}{R_{c}}+\frac{V_{c}(\omega, t)}{Z_{0} n^{2}}=\frac{I_{g}}{n}$.

Analytic solutions including dissipated power ( $\left.P_{d}=\frac{V_{c}^{2}}{2 R_{c}}\right)$, stored energy change $\left(P_{s}=\frac{d U}{d t}\right)$, reflected power ( $\left.P_{r}=P_{i n}-P_{d}-P_{s}\right)$, and emitted power ( $P_{e}=\frac{V_{c}^{2}}{2 Z_{0} n^{2}}$ ) for the pulse length of $1300 \mu \mathrm{s}$ and the loaded quality factor of $7.6 \mathrm{e}+5$ are graphically shown in Fig. 6. To maintain a $14.5 \mathrm{MV} / \mathrm{m}$ accelerating gradient without beam loading, the required power from the generator is set to $11 \mathrm{~kW}$ at this loaded quality factor. At first, most power is reflected during the filling stage of the cavity. After then, the reflected power will pass through the zero and reaches its nonzero steady-state level (11 $\mathrm{kW}$ ) [6]. When the klystron is abruptly turned off, the reflected power is just equal to the emitted power and exponentially decreased to zero.

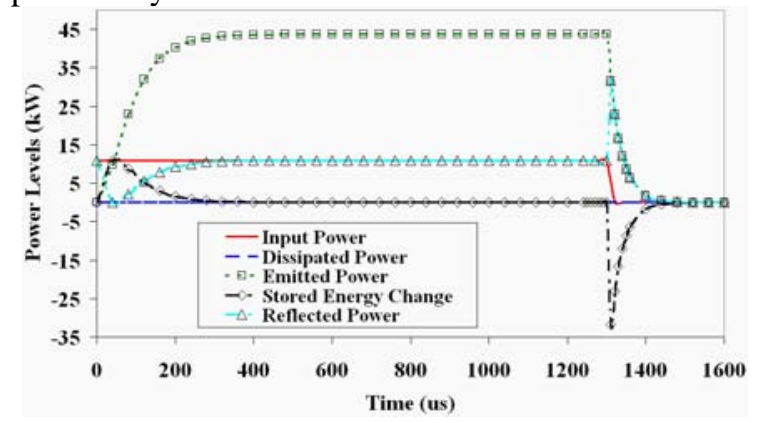

Figure 6: System power response versus the time.

\section{Results with Beam Loading}

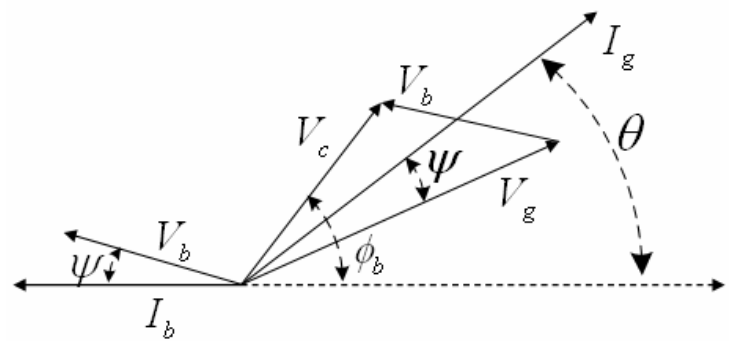

Figure 7: Vector diagram of the generator-induced voltage and the beam-induced voltage in a detuned cavity.

A current source in parallel with the generator in the equivalent circuit model can be simply represented the beam current based on a series of ideal assumptions [6]. It is useful and important to investigate the required power from the generator for the coupling system with relation to the loaded quality factor and the beam current. Solutions can be more easily derived from the vector diagram [8] shown in Fig. 7 than from the circuit differential equation. Some definitions are mentioned in advance. $P_{g}$ is the required power from the generator, $V_{c}$ is the cavity voltage, $I_{b 0}$ denotes the DC beam current, $\phi_{b}$ is the beam delay phase, $Q_{L}$ is the loaded quality 
factor, and $\tan \psi$ denotes the detuning angle, respectively.

It is easy to write the two equations [8]:

$$
\begin{aligned}
& V_{c}=V_{g} \cos \left(\phi_{b}-\theta+\psi\right)-V_{b} \cos \left(\psi+\phi_{b}\right) \\
& 0=V_{g} \sin \left(\phi_{b}-\theta+\psi\right)-V_{b} \sin \left(\psi+\phi_{b}\right)
\end{aligned}
$$

Try to cancel $\cos \left(\phi_{b}-\theta+\psi\right), \sin \left(\phi_{b}-\theta+\psi\right)$ and use the following equations [8]:

$$
\left(\frac{R}{Q_{0}}\right) Q_{L}=\frac{V_{c}^{2}}{w U} \frac{w U}{\left(\frac{V_{c}^{2}}{2 R_{L}}\right)}=2 R_{L}, V_{g}=\sqrt{\frac{8 \beta R_{L} P_{g}}{\beta+1}} \cos \psi,
$$

and $V_{b}=R_{L} I_{b} \cos \psi=2 R_{L} I_{b 0} \cos \psi$.

Finally, we get (for heavy beam loading, $\beta>1$ ):

$P_{g}=\frac{V_{c}^{2}}{4\left(\frac{R}{Q}\right) \cdot Q_{L}}\left\{\left(1+\frac{\left(\frac{R}{Q}\right) \cdot Q_{L} I_{b 0}}{V_{c}} \cos \phi_{b}\right)^{2}+\left(\tan \psi-\frac{\left(\frac{R}{Q}\right) \cdot Q_{L} \cdot I_{b 0}}{V_{c}} \sin \phi_{b}\right)^{2}\right\}$.

The required power from the generator for different loaded quality factors and beam currents are shown in Fig. 8 and Table 3.

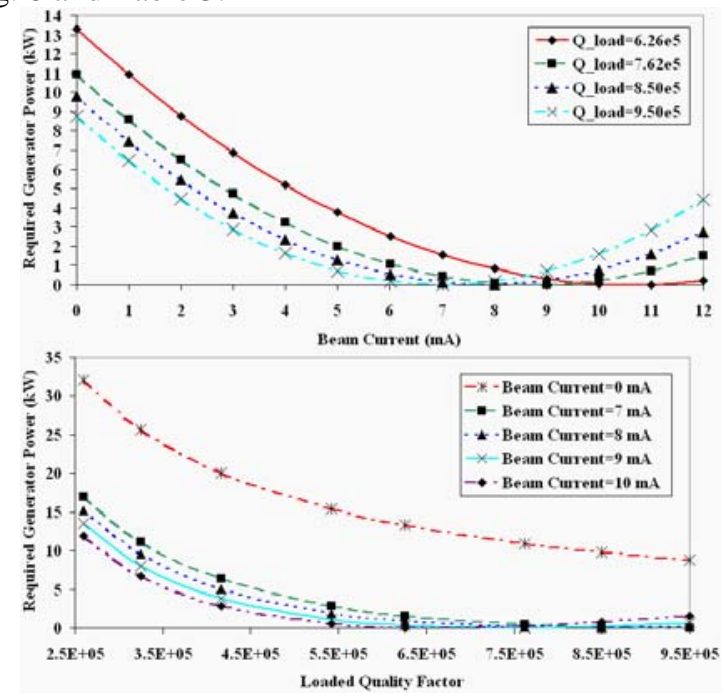

Figure 8: Required generator power as a function of the beam current and the loaded quality factor.

Table 3: Required generator power with relation to the loaded quality factor and the beam current. *The phase of the incident wave is changed of $180^{\circ}$.

\begin{tabular}{|l|l|l|l|l|l|}
\hline \multirow{2}{*}{$\begin{array}{l}\text { Generator } \\
\text { Power }(\mathbf{k W})\end{array}$} & \multicolumn{5}{|c|}{ Loaded Quality Factor } \\
\cline { 2 - 6 } & $\mathbf{6 . 2 6 e 5}$ & $\mathbf{7 . 6 2 e 5}$ & $\mathbf{8 . 5 0 e 5}$ & $\mathbf{9 . 5 0 e 5}$ \\
\hline \multirow{3}{*}{} & $\mathbf{0}$ & 13.31 & 10.94 & 9.80 & 8.77 \\
\cline { 2 - 6 } & $\mathbf{7}$ & 1.57 & 0.44 & 0.12 & 0 \\
\cline { 2 - 6 } & $\mathbf{8}$ & 0.83 & 0.08 & 0 & $0.17^{*}$ \\
\cline { 2 - 6 } & $\mathbf{9}$ & 0.32 & 0 & $0.22^{*}$ & $0.70^{*}$ \\
\cline { 2 - 6 } & $\mathbf{1 0}$ & 0.05 & $0.23^{*}$ & $0.75^{*}$ & $1.59^{*}$ \\
\cline { 2 - 6 } & $\mathbf{1 1}$ & 0.02 & $0.73^{*}$ & $1.59^{*}$ & $2.83^{*}$ \\
\cline { 2 - 6 } & $\mathbf{1 2}$ & $0.22^{*}$ & $1.51^{*}$ & $2.76^{*}$ & $4.43^{*}$ \\
\hline
\end{tabular}

From Table 3 , we find that the klystron can be turned off when the beam $(9 \mathrm{~mA})$ is induced into the system if the loaded quality factor is set to $7.6 \mathrm{e}+5$, i.e., the antenna is flush with the wall of the beam tube. Here beam can be looked as an energy source proving $45 \mathrm{~kW}$ RF power for the cavity ( $V_{c}=5 M V$ ) at a given beam current of $9 m A$. Even if the loaded quality factor is equal to $9.5 \mathrm{e}+5$, we only need $0.7 \mathrm{~kW}$ generator power. Most power to supply the system is acquired from the beam.

\section{CONCLUSION}

Our design of the third harmonic coupling system can couple the proper amount of power to the cavity and the beam and guarantee the minimum power consumption from the generator. The klystron can even be turned off under some special conditions. The coupling system can be easily adapted to meet the requirements of different scenario applications through adjusting the antenna penetration length or the generator power level.

\section{ACKNOWLEDGEMENTS}

We would like to thank Markus Huening and WolfDietrich Moeller from DESY for helpful discussions and Daniel Olis from Fermilab for the supply of the geometry data.

\section{REFERENCES}

[1] N. Solyak et al., "The Progress in Developing Superconducting Third Harmonic Cavity," Proceedings of EPAC 2006, Edinburgh, Scotland, June 2006, p. 804-806.

[2] N. Solyak et al., “3.9 GHz Superconducting Accelerating 9-cell Cavity Vertical Test Results,” Proceedings of PAC 2007, Albuquerque, NM, June 2007, WEPMN111.

[3] E. Harms et al., "Status of the 3.9-GHz Superconducting RF Cavity Technology at Fermilab,” Proceedings of PAC 2007, Albuquerque, NM, June 2007, WEPMN096.

[4] J. Li et al., "Simulations and Optimizations of a New Power Coupler for 3.9-GHZ Superconducting Cavities at Fermilab,” Proceedings of LINAC 2006, Knoxville, TN, August 2006, p. 701-703.

[5] J. Li et al., "RF Design and Processing of a Power Coupler for Third Harmonic Superconducting Cavities,” Proceedings of PAC 2007, Albuquerque, NM, June 2007, WEPMN100.

[6] H. Padamsee, J. Knobloch, and T. Hays, "RF Superconductivity for Accelerators,” John Wiley \& Sons, INC, 1998.

[7] A. Sun, "Measurement Formulae for a Superconducting RF Cavity without Beam Load," SNS-NOTE-AP-135, December 102004.

[8] T. Schilcher, "Vector Sum Control of Pulsed Accelerating Fields in Lorentz Force Detuned Superconducting Cavities,” Dissertation, Hamburg, Germany, 1998. 